\title{
EULAR recommendations for the use of imaging in large vessel vasculitis in clinical practice
}

\author{
Christian Dejaco, ${ }^{1,2}$ Sofia Ramiro, ${ }^{3}$ Christina Duftner, ${ }^{4}$ \\ Florent L Besson, ${ }^{5,6}$ Thorsten A Bley, ${ }^{7}$ Daniel Blockmans, ${ }^{8}$ Elisabeth Brouwer, ${ }^{9}$ \\ Marco A Cimmino, ${ }^{10}$ Eric Clark, ${ }^{11}$ Bhaskar Dasgupta, ${ }^{12,13}$ Andreas P Diamantopoulos, ${ }^{14}$ \\ Haner Direskeneli, ${ }_{15}^{15}$ Annamaria lagnocco, ${ }^{16}$ Thorsten Klink, ${ }^{7}$ Lorna Neill, ${ }^{17}$ \\ Cristina Ponte, ${ }^{18,19}$ Carlo Salvarani, $^{20,21}$ Riemer H J A Slart, ${ }^{22,23}$ Madeline Whitlock, ${ }^{12}$ \\ Wolfgang A Schmidt ${ }^{24}$
}

\begin{abstract}
Handling editor Josef S Smolen

For numbered affiliations see end of article.
\end{abstract}

\section{Correspondence to}

Dr Christian Dejaco, Department of Rheumatology and Immunology, Medical University Graz, Graz 8036, Austria; christian.dejaco@gmx.net

Received 4 November 2017 Revised 13 December 2017 Accepted 2 January 2018

Published Online First

22 January 2018
Check for updates

To cite: Dejaco C, Ramiro S, Duftner $C$, et al. Ann Rheum Dis 2018;77:636-643.

\section{ABSTRACT}

To develop evidence-based recommendations for the use of imaging modalities in primary large vessel vasculitis (LVV) including giant cell arteritis (GCA) and Takayasu arteritis (TAK). European League Against Rheumatism (EULAR) standardised operating procedures were followed. A systematic literature review was conducted to retrieve data on the role of imaging modalities including ultrasound, MRI, CT and $\left[{ }^{18} \mathrm{~F}\right]$-fluorodeoxyglucose positron emission tomography (PET) in LVV. Based on evidence and expert opinion, the task force consisting of 20 physicians, healthcare professionals and patients from 10 EULAR countries developed recommendations, with consensus obtained through voting. The final level of agreement was voted anonymously. A total of 12 recommendations have been formulated. The task force recommends an early imaging test in patients with suspected LVV, with ultrasound and MRI being the first choices in GCA and TAK, respectively. CT or PET may be used alternatively. In case the diagnosis is still in question after clinical examination and imaging, additional investigations including temporal artery biopsy and/or additional imaging are required. In patients with a suspected flare, imaging might help to better assess disease activity. The frequency and choice of imaging modalities for long-term monitoring of structural damage remains an individual decision; close monitoring for aortic aneurysms should be conducted in patients at risk for this complication. All imaging should be performed by a trained specialist using appropriate operational procedures and settings. These are the first EULAR recommendations providing up-to-date guidance for the role of imaging in the diagnosis and monitoring of patients with (suspected) LVV.

\section{INTRODUCTION}

Large vessel vasculitis (LVV) is the most common form of primary vasculitis comprising giant cell arteritis (GCA) and Takayasu arteritis (TAK). ${ }^{12}$ The field of GCA and LVV has undergone rapid expansion. Ultrasound-guided fast-track strategies have led to a reduction of irreversible vision loss, and the concept of imaging confirmed large vessel (LV-)GCA with or without cranial disease, has been added to the disease definition. ${ }^{3-5}$ Based on these considerations, the importance of imaging modalities including ultrasound, MRI, CT and $\left[{ }^{18} \mathrm{~F}\right]$-fluorodeoxyglucose positron emission tomography (PET) has steadily increased. ${ }^{6}$ These techniques enable the assessment of cranial and extracranial arteries and the aorta and are less invasive, more sensitive and more quickly available than temporal artery biopsy (TAB) and conventional angiography, which have been the sole diagnostic standards in GCA and TAK, respectively, for decades.

In TAK, temporal arteries are usually spared, and extracranial artery biopsies are rarely feasible. Angiography visualises luminal changes caused by vasculitis such as stenosis or occlusion but cannot delineate vessel wall pathology. Besides, angiography bears the risk of allergic reactions, haematoma, iatrogenic embolisation and arterial dissection. Modern imaging methods have therefore almost replaced catheterised angiography unless it is performed for therapeutic vascular interventions. ${ }^{8}$

These advances have brought along significant controversy and uncertainty about when to use which imaging technique, whether imaging might be helpful during follow-up to assess disease activity and damage and whether imaging results might predict future outcomes.

The objective of this project was to provide userfriendly, evidence-based recommendations for the use of imaging modalities for diagnosis, monitoring and outcome prediction of primary LVV.

\section{METHODS}

After approval by the EULAR Executive Committee, the convenors (ChristiaD and WAS) and methodologist (SR) led a task force guided by the 2014 updated EULAR standardised operating procedures. $^{9}$ The 20 task force members consisted of rheumatologists, radiologists, nuclear medicine specialists, patient representatives, an internist, a methodologist, a healthcare professional and EMerging EUlar NETwork representatives from 10 countries. All members disclosed their potential conflicts of interest before the start of the process. Two task force meetings took place.

At the first task force meeting, the panel agreed on four key questions covering the following aspects: the role of imaging techniques (including ultrasound, MRI, CT and PET) in (1) diagnosis and (2) monitoring of inflammation and damage, (3) prediction of outcome and (4) required technical standards for imaging. 
Table 1 Oxford Centre for Evidence-Based Medicine 2011 levels of evidence for diagnostic studies (modified according to ref 13)

\begin{tabular}{ll}
\hline Level & Definition \\
\hline 1 & $\begin{array}{l}\text { Evidence from a systematic review of cross-sectional studies with } \\
\text { consistently applied reference standard and blinding. }\end{array}$ \\
\hline 2 & $\begin{array}{l}\text { Individual cross-sectional studies with consistently applied reference } \\
\text { standard and blinding. }\end{array}$ \\
\hline 3 & $\begin{array}{l}\text { Non-consecutive studies or studies without consistently applied reference } \\
\text { standards. }\end{array}$ \\
\hline 5 & Case-control studies or poor or non-independent reference standard. \\
\hline $\begin{array}{l}\text { Level of evidence manism-based reasoning. } \\
\text { indirectness, because of inconsistency between studies or because the absolute } \\
\text { effect size is very small. Level may be upgraded if there is a large or very large } \\
\text { effect size. }\end{array}$
\end{tabular}

The systematic literature review (SLR) was conducted by two task force members (ChristinD and ChristiaD) under the guidance of the methodologist. Only prospective studies conducted in $>20$ patients with suspected and/or established primary LVV were included. The evidence summarised in the SLR was presented to the task force in the form of tables summarising the findings, including an assessment of the risk of bias of the studies. ${ }^{1011}$ The SLR is published separately ${ }^{12}$; however, the SLR and the present recommendations manuscript form an integral and inseparable part and should be read as such.

At the second meeting, the task force formulated the recommendations based on the evidence and expert opinion in a process of discussion and consensus, followed by final voting on the recommendations. Consensus was accepted if $>75 \%$ of the members voted in favour of the recommendation at the first round, $>67 \%$ at the second round and at a third round $>50 \%$ was accepted. The Oxford Centre for EvidenceBased Medicine levels of evidence (LoE) derived from the SLR were added to each recommendation (table 1). ${ }^{13}$

Finally, each task force member anonymously indicated the level of agreement via e-mail (LoA, 0-10 numeric rating scale with $0=$ do not agree and $10=$ fully agree). The mean and SD of the LoA, as well as the percentage of task force members with an agreement $\geq 8$ are presented.

Based on the gaps in the evidence and the issues of controversy, a research agenda was formulated. The final manuscript was reviewed and approved by all task force members and approved by the EULAR Executive Committee.

\section{RESULTS}

\section{General aspects}

These recommendations are intended to advise physicians on the use of imaging modalities (including ultrasound, MRI, CT and PET) when making a clinical diagnosis of LVV and when to apply imaging for monitoring of disease activity and damage. CT and MRI also refer to specific angiography techniques such as CT angiography (CTA) and MR angiography (MRA), and PET is commonly used in conjunction with CT or CTA.

These recommendations are not intended to cover all aspects of diagnosis and management of LVV and particularly do not discuss in full the role of TAB for GCA diagnosis.

The targeted users of these recommendations are secondary and tertiary care physicians including rheumatologists, ophthalmologists, neurologists, radiologists, nuclear medicine specialists, vascular surgeons, angiologists, geriatricians and other specialists in general (internal) medicine. The target population is patients with suspected or established primary LVV, specifically
GCA or TAK. These recommendations may also inform patients participating in shared decision making, primary care physicians and healthcare providers organising care of patients with LVV.

\section{Recommendations}

A total of 12 recommendations have been formulated that are summarised in table 2 (including the LoE and LoA) and are discussed in detail below.

Recommendation 1: in patients with suspected GCA, an early imaging test is recommended to complement the clinical criteria for diagnosing GCA, assuming high expertise and prompt availability of the imaging technique. Imaging should not delay initiation of treatment.

This recommendation is general in its nature and intended to provide a framework for the subsequent specific recommendations on different imaging modalities. The choice of the individual imaging method depends on the predominant clinical symptoms and local settings as specified below. Early confirmation or exclusion of GCA by a diagnostic test is essential in order to prevent disease complications such as blindness or toxicity from unnecessary treatment. ${ }^{14}$ The task force members recognised that many physicians still consider $\mathrm{TAB}$ as the gold standard test for the diagnosis of GCA. The present (and subsequent) proposition(s) should not be understood as a recommendation against performing $\mathrm{TAB}$. In settings where imaging modalities are not readily available or expertise with imaging in GCA is questionable, a biopsy should still be favoured in first place. Besides, if positive histology is already available, additional imaging may not be needed for the diagnosis. In centres, however, where imaging (and TAB) is readily available and performed with high quality, the task force recommends that imaging should be preferred as the first test because of low invasiveness, ready availability of imaging results and assessment of a larger extent of potentially inflamed arteries at the same examination, thus contributing to a lower number of false negative results.

Imaging should be performed before or as early as possible after initiation of therapy, best within 1 week, because treatment with glucocorticoids rapidly reduces the sensitivity of imaging. ${ }^{15-18}$ Treatment, however, should never be delayed in patients with a strong suspicion of GCA due to outstanding imaging or other diagnostic tests, because ischaemic complications such as blindness occur almost exclusively before initiation of therapy. ${ }^{14}$

The procedural risk of TAB is low; however, there is burden to patients and resource use. ${ }^{19}$ Ultrasound in all patients with suspected GCA has been reported as cost-effective compared with biopsy plus clinical judgement alone with a net monetary benefit of $£ 485$ ( $€ \sim 550 /$ US $\$ \sim 600)$ per patient. ${ }^{15}$ Modelling of cost-effectiveness analysis considered the costs of the tests as well as the consequences of correct and incorrect diagnosis resulting in drug toxicity or vision loss that might have been prevented by one or the other test. Marking arterial segments with ultrasound to guide subsequent biopsy failed to increase the sensitivity of $\mathrm{TAB}$ in one randomised study ${ }^{20}$; however, additional research is necessary to better investigate this issue.

In patients with predominately LV-GCA, a lower sensitivity of TAB has been reported as compared with cranial GCA. ${ }^{21-23}$ As TAB has not been conducted systematically in these studies, future studies should be conducted to investigate the diagnostic value of TAB for LV-GCA.

Recommendation 2: in patients in whom there is a high clinical suspicion of GCA and a positive imaging test, the diagnosis 
Table 2 EULAR recommendations for the use of imaging in LVV in clinical practice

\begin{tabular}{|c|c|c|}
\hline Statement & LoE & LoA \\
\hline $\begin{array}{l}\text { 1. In patients with suspected GCA, an early imaging test is recommended to complement the clinical criteria for diagnosing GCA, } \\
\text { assuming high expertise and prompt availability of the imaging technique. Imaging should not delay initiation of treatment. }\end{array}$ & 1 & $\begin{array}{l}9.2(2.1) \\
90 \% \geq 8\end{array}$ \\
\hline $\begin{array}{l}\text { 2. In patients in whom there is a high clinical suspicion of GCA and a positive imaging test, the diagnosis of GCA may be made without } \\
\text { an additional test (biopsy or further imaging). In patients with a low clinical probability and a negative imaging result, the diagnosis of } \\
\text { GCA can be considered unlikely. In all other situations, additional efforts towards a diagnosis are necessary. }\end{array}$ & 2 & $\begin{array}{l}9.4(1.0) \\
90 \% \geq 8\end{array}$ \\
\hline $\begin{array}{l}\text { 3. Ultrasound of temporal } \pm \text { axillary arteries is recommended as the first imaging modality in patients with suspected predominantly } \\
\text { cranial GCA*. A non-compressible 'halo' sign is the ultrasound finding most suggestive of GCA. }\end{array}$ & 1 & $\begin{array}{l}9.7(0.6) \\
100 \% \\
\geqq 8\end{array}$ \\
\hline 5. CT† and PET† are not recommended for the assessment of inflammation of cranial arteries. & 5 & $\begin{array}{l}9.5(1.2) \\
95 \%>8\end{array}$ \\
\hline $\begin{array}{l}\text { 6. Ultrasound, PET, MRI and/or CT may be used for detection of mural inflammation and/or luminal changes in extracranial arteries to } \\
\text { support the diagnosis of LV-GCA. Ultrasound is of limited value for assessment of aortitis. }\end{array}$ & $\begin{array}{l}3 \text { (PET and } \mathrm{CT} \text { ) and } 5 \\
\text { (MRI and ultrasound) }\end{array}$ & $\begin{array}{l}9.8(0.6) \\
100 \% \\
\geqq 8\end{array}$ \\
\hline $\begin{array}{l}\text { 9. Conventional angiography is not recommended for the diagnosis of GCA or TAK as it has been superseded by the previously } \\
\text { mentioned imaging modalities. }\end{array}$ & 5 & $\begin{array}{l}9.8(0.6) \\
100 \% \geq 8\end{array}$ \\
\hline $\begin{array}{l}\text { 10. In patients with LVV (GCA or TAK) in whom a flare is suspected, imaging might be helpful to confirm or exclude it. Imaging is not } \\
\text { routinely recommended for patients in clinical and biochemical remission. }\end{array}$ & 5 & $\begin{array}{l}9.4(0.8) \\
100 \% \geq 8\end{array}$ \\
\hline $\begin{array}{l}\text { 11. In patients with LVV (GCA or TAK), MRA, CTA and/or ultrasound may be used for long-term monitoring of structural damage, } \\
\text { particularly to detect stenosis, occlusion, dilatation and/or aneurysms. The frequency of screening as well as the imaging method } \\
\text { applied should be decided on an individual basis. }\end{array}$ & 5 & $\begin{array}{l}9.3(1.2) \\
95 \% \geq 8\end{array}$ \\
\hline $\begin{array}{l}\text { 12. Imaging examination should be done by a trained specialist using appropriate equipment, operational procedures and settings. The } \\
\text { reliability of imaging, which has often been a concern, can be improved by specific training. Suggestions for technical and operational } \\
\text { parameters are depicted in box } 1 \text {. }\end{array}$ & 5 & $\begin{array}{l}9.8(0.6) \\
100 \% \\
\geq 8\end{array}$ \\
\hline
\end{tabular}

Numbers in column 'LoA' indicate the mean and SD (in parentheses) of the LoA, as well as the percentage of task force members with an agreement $\geq 8$.

${ }^{*}$ Cranial symptoms of GCA include headache, visual symptoms, jaw claudication, swelling and/or tenderness of temporal arteries.

†CT and MRI also refers to specific angiography techniques such as CT angiography (CTA) and MR angiography (MRA), and PET is commonly combined with CT or CTA.

$\ddagger$ Cranial arteries: superficial temporal, occipital and facial, usually all visible in one examination in MRI.

EULAR, European League Against Rheumatism; GCA, giant cell arteritis; LoA, level of agreement; LoE, level of evidence; LV-GCA, large vessel GCA; LVV, large vessel vasculitis;

PET, positron emission tomography; TAK, Takayasu arteritis.

of GCA may be made without an additional test (biopsy or further imaging). In patients with a low clinical probability and a negative imaging result, the diagnosis of GCA can be considered unlikely. In all other situations, additional efforts towards a diagnosis are necessary.

The performance of a diagnostic test depends on its sensitivity and specificity and on the clinical situation where it is applied, that is, on the particular pretest probability. ${ }^{24}$ For example, a patient with 50 years of age, with chronic unspecific headache and normal inflammatory markers has a very low pretest clinical probability for the presence of GCA. Assuming a pretest probability of $5 \%$ and a positive ultrasound result (which has a $77 \%$ sensitivity and a $96 \%$ specificity), ${ }^{12}$ the post-test probability would increase to $50 \%$ only. ${ }^{24}$ In case of a negative test, however, the diagnosis of GCA is very unlikely with a post-test probability of $1.3 \%$. In patients with a high clinical suspicion of GCA (>50\%), for example, in case of new-onset headache, visual symptoms, jaw claudication and elevated erythrocyte sedimentation rate (ESR) and $\mathrm{C}$ reactive protein, a positive ultrasound would result in a post-test probability of $>95 \%$. A negative examination decreases the probability to $20 \%$, hence, GCA is still a possible option and further investigation is necessary. In clinical practice, the pretest probability needs to be determined case by case since a clinical probability score, as it has been published for other diseases, ${ }^{25}$ is not yet available for GCA. Estimating the pretest probability for predominately
LV-GCA might be particularly challenging because symptoms of LV-GCA are often vague.

The task force clearly emphasised that in all cases, where GCA cannot be confirmed or excluded based on clinical, laboratory and imaging results, every effort towards a diagnosis should be made including additional tests such as TAB and/or additional imaging.

Recommendation 3: ultrasound of temporal \pm axillary arteries is recommended as the first imaging modality in patients with suspected predominantly cranial GCA. ${ }^{\mathrm{i}}$ A non-compressible 'halo' sign is the ultrasound finding most suggestive of GCA.

Ultrasound should be the primary imaging test in patients with suspected GCA presenting predominantly with cranial symptoms because of a high LoE of good test performance, easy access, absence of radiation or other procedural risks and the relative low costs as compared with other modalities.

The 'halo' sign of temporal arteries is the most relevant ultrasound finding in GCA. Recently, it has been defined by an Outcome Measures in Rheumatology (OMERACT) working group as a 'homogenous, hypoechoic wall thickening that is well delineated towards the luminal side that is visible both in longitudinal and transverse planes, most commonly concentric in

${ }^{\mathrm{i} C r a n i a l}$ symptoms of GCA include headache, visual symptoms, jaw claudication, swelling and/or tenderness of temporal arteries. 
transverse scans'. ${ }^{26}$ The 'halo' sign at temporal arteries revealed a pooled sensitivity of $77 \%$ and a pooled specificity of $96 \%$ as compared with the clinical diagnosis of GCA. ${ }^{12}$ These values remained consistent in a series of sensitivity analyses. ${ }^{12}$ The persistence of a hypoechoic swelling despite the compression of the artery lumen with the ultrasound probe (termed 'compression' sign) is a variant of the 'halo' sign revealing sensitivities of $77 \%-79 \%$ and a specificity of $100 \% .^{27} 28$ The detection of temporal artery stenosis or occlusion did not increase the diagnostic yield over the halo sign alone.

False-positive halos might occasionally be detected in other forms of vasculitis (eg, in ANCA-associated vasculitis), in infectious diseases or in patients with (severe) arteriosclerosis. ${ }^{29-31}$ Ultrasound results should therefore always be interpreted together with clinical and laboratory findings as stated above.

According to expert opinion, examination of axillary arteries is particularly helpful in patients with suspected GCA but negative or inconclusive temporal artery ultrasound. The SLR revealed only a slight increment in the sensitivity $(2 \%)$ in one study that considered the axillary arteries as compared with the assessment of temporal arteries alone. ${ }^{31}$ The recommendation of the task force is therefore to primarily investigate the temporal arteries. Where this examination is non-diagnostic and a clinical suspicion of GCA remains, additional vessels such as axillary or other cranial and/or extracranial arteries should be scanned.

Recommendation 4: high resolution MRI of cranial arteries ${ }^{\mathrm{ii}}$ to investigate mural inflammation may be used as an alternative for GCA diagnosis if ultrasound is not available or inconclusive.

High resolution MRI of superficial cranial arteries should be considered as an alternative to ultrasound. The diagnostic value of both modalities is comparable (pooled sensitivity of MRI: $73 \%$; specificity: $88 \%) .{ }^{12}$ Similarly, a retrospective direct comparison of MRI and ultrasound revealed a similar sensitivity (69\% and 67\%, respectively) and specificity (both 91\% and 91\%) of both techniques. ${ }^{32}$

The main limitations of MRI are restricted availability, costs and possible adverse effects of contrast agents. MRI might only be feasible if emergency referrals for GCA can be implemented. It is strongly advised not to delay GC therapy due to outstanding imaging, and MRI of cranial arteries needs to be performed immediately within the first days of GC therapy in order to avoid false-negative results.

The advantages of MRI over ultrasound are a higher standardisation of data acquisition and the possibility to investigate multiple cranial and extracranial arteries including the aorta at the same time, which might reduce the probability of missing inflammation in case of skip lesions. This requires specific technical settings with multiple coils and a long time on the MRI scanner, which is not always feasible. MRI can also assess intracranial arteries, which may be affected in GCA. Other intracranial vasculopathies such as primary cerebral angiitis, atherosclerosis or reversible cerebral vasoconstriction syndrome must be differentiated from an intracranial manifestation of GCA. In a small prospective study, the examination of intracranial arteries did not increase the diagnostic yield for $\mathrm{GCA}^{33}$; however, further studies are needed to investigate this issue.

${ }^{\text {ii } C r a n i a l ~ a r t e r i e s: ~ s u p e r f i c i a l ~ t e m p o r a l, ~ o c c i p i t a l ~ a n d ~ f a c i a l, ~ u s u a l l y ~ a l l ~}$ visible in one examination in MRI.
Recommendation 5: CT and PET are not recommended for the assessment of inflammation of cranial arteries.

The task force does not recommend CT or PET for the assessment of cranial arteries because of lack of evidence, radiation exposure and high resource use. No studies have been conducted on these imaging modalities for the assessment of cranial arteries in GCA. ${ }^{12}$ The use of PET is limited by the proximity of the brain; hence, superficial cranial vessels cannot be distinguished from the brain.

Recommendation 6: ultrasound, PET, MRI and/or CT may be used for detection of mural inflammation and/or luminal changes in extracranial arteries to support the diagnosis of LV-GCA. Ultrasound is of limited value for assessment of aortitis.

This recommendation is mainly based on expert opinion. The best imaging technique for patients with suspected LV-GCA and predominantly systemic symptoms is unclear and depends on local settings and expertise. While ultrasound has advantages as outlined above, it has limited access to the thoracic aorta. Besides, the exact sensitivity and specificity of ultrasound for LV-GCA is unknown because this subgroup of patients has not been analysed separately in ultrasound studies. ${ }^{3134}$

The major advantage of PET in patients with systemic symptoms is the ability to identify GCA along with other serious pathology such as infections or tumours. This may be particularly relevant in elderly patients with constitutional symptoms without specific clinical features of GCA and/or PMR. Two clinical studies reported divergent sensitivities (67\%-77\%) and specificities (66\%-100\%) for PET, which may be explained by the small sample size, the lack of independence between index test and the reference standard, use of GC as well as the circular application of different criteria for GCA. ${ }^{35} 36$ Disadvantages of PET are high costs, lower availability and radiation exposure. Inexperienced readers may misinterpret atherosclerosis as LVV. ${ }^{37}$ Missing information on wall-thickness and luminal changes can be overcome by combining PET with CT.

The advantages of MRI are the absence of radiation and the contemporaneous detection of structural lesions (such as vessel wall thickening and luminal stenosis/occlusion) and contrast enhancement of the arterial wall, which is presumed (but not proven) to reflect active inflammation. Specific sequences are required to image both the arterial wall and the arterial lumen as outlined in box 1 . The SLR did not retrieve any study investigating the use of MRI in LV-GCA. ${ }^{12}$

CT may also be useful to detect structural lesions and wall inflammation and enables a higher resolution and shorter procedural time than MRI; however, this is at the cost of radiation exposure. Evidence from literature is scarce with only a single small study indicating a sensitivity of $73 \%$ and a specificity of $78 \%$ of CTA for the diagnosis of LV-GCA. ${ }^{35}$

Recommendation 7: in patients with suspected TAK, MRI to investigate mural inflammation and/or luminal changes should be used as the first imaging test to make a diagnosis of TAK, assuming high expertise and prompt availability of the technique.

This recommendation is almost entirely based on expert opinion and current clinical practice. A technique without radiation exposure is preferable over other modalities because of the young age of patients with TAK. Besides, MRI enables assessment of the vessel wall and luminal changes, which are both relevant for TAK. In one study, MRA yielded a sensitivity and specificity of $100 \%$ for TAK using conventional angiography as the reference standard. ${ }^{38}$ The most important limitation of MRI is the restricted availability as compared with ultrasound or CT. 


\section{Ultrasound}

- High-quality, modern equipment is essential. Linear probes are recommended for supra-aortic arteries, sector or convex probes for ascending aorta and aortic arch and convex probes for abdominal aorta. Settings may slightly vary according to different equipment.

- The B-mode frequency should be $\geq 15 \mathrm{MHz}$ for temporal arteries and 7-15 MHz for extracranial supra-aortic arteries. Image depth should be $10-20 \mathrm{~mm}$ for temporal arteries and $30-40 \mathrm{~mm}$ for extracranial supra-aortic arteries.

- The focus should be at the level of the artery. The B-mode gain should be adjusted to avoid anechoic appearance of the artery wall. The colour Doppler gain should be adjusted to avoid underfilling or overfilling of the vessel lumen.

- Colour Doppler mode is preferred over power Doppler mode. Tissue harmonic imaging may improve delineation of the intima-media complex.

- Doppler frequencies of 7-12 MHz and 4-8 MHz should be applied for the temporal and for the extracranial supra-aortic arteries, respectively. PRF should be $2-3.5 \mathrm{kHz}$ and $3-4 \mathrm{kHz}$, respectively. The angle between sound waves and artery should be $\leq 60^{\circ}$.

\section{CT}

- Multislice CT scanner should be used.

- Collimation $0.6 \mathrm{~mm}$, tube voltage $120 \mathrm{kV}$, tube current time product (mAs) determined by automatic dose modulation.

- Reconstruction slice thickness should be between $0.5 \mathrm{~mm}$ and $1.0 \mathrm{~mm}$.

- Body-weight adapted injection of $60-120 \mathrm{~mL}$ of non-ionic iodinated contrast agent ( $\geq 350 \mathrm{mg} / \mathrm{mL}$ ) using a power injector ( $\geq 4 \mathrm{~mL} / \mathrm{s})$.

- Arterial phase: bolus-tracking method (threshold of $100 \mathrm{HU}$ ); ECG triggering.

- Venous phase: $50 \mathrm{~s}$ after finishing the arterial phase acquisition.

\section{MRI}

Cranial MRI technique:

- 1.5 T, preferentially 3.0 T MRI scanner, minimum 8-channel head-coil.

- T1-weighted spin echo, gadolinium contrast-enhanced, fat-suppressed, high-resolution (inplane $<<1 \mathrm{~mm}^{2}$, for example, 195×260 $\mu \mathrm{m}$, slice thickness $3 \mathrm{~mm}$, repetition time (TR)/echo time (TE) 500/22 ms).

- T2-weighted turbo spin echo (TSE), non-contrast-enhanced imaging (TR/TE 9000/143 ms) is significantly less sensitive.

- Transversal slices angulated parallel to skull base.

Body MRI technique:

- 1.5 T, preferentially 3.0 T MRI scanner, minimum 8-channel head and neck coil and 16-channel body coil.

- MR angiography of aorta and major branches from carotid bifurcation to iliac arteries in coronal acquisition to include axillary and brachial arteries $\rightarrow$ detection of vessel lumen (stenosis, occlusion and aneurysm).

- T1-weighted, fat-suppressed, contrast-enhanced, black blood imaging (eg, navigated three-dimensional TSE, spatial resolution $1.2 \times 1.3 \times 2 \mathrm{~mm}^{3}$, TR/TE 1000/35 ms) $\rightarrow$ assessment of mural inflammation.

- T2-weighted TSE imaging for oedema detection in mural inflammation is less sensitive and more prone to artefacts.

\section{$\left[{ }^{18} \mathrm{~F}\right]$-Fluorodeoxyglucose (FDG) positron emission tomography (PET)}

- Hybrid PET with low-dose CT.

- Blood glucose levels: preferred $<7 \mathrm{mmol} / \mathrm{L}(126 \mathrm{mg} / \mathrm{dL}),<10 \mathrm{mmol} / \mathrm{L}(180 \mathrm{mg} / \mathrm{dL})$ acceptable.

- Interval between FDG infusion and image acquisition should be at least $60 \mathrm{~min}$, preferably $90 \mathrm{~min}$.

- Position of patient is supine, position of the arms should be arms down.

- Body parts to include: from top of head to at least midthigh, preferably to below the knees.

- Scoring FDG uptake: qualitative visual grading; if result is unclear, compare it with the liver background (grading 0-3).

Recommendation 8: PET, CT and/or ultrasound may be used as alternative imaging modalities in patients with suspected TAK. Ultrasound is of limited value for assessment of the thoracic aorta.

This recommendation is also based on expert opinion. The task force felt that PET might be particularly valuable in patients with unspecific symptoms to detect alternative causes of illness. CT (also in conjunction with PET) enables visualisation of vessel wall and luminal changes and is widely available. However, it is associated with significant radiation. Only a single small study was available for CTA yielding a sensitivity and specificity of $100 \%$ for the diagnosis of TAK using conventional angiography as the reference standard. ${ }^{39}$ No studies were available for PET and for ultrasound. Ultrasound might be particularly valuable in patients with claudication of upper and/or lower limbs.
Recommendation 9: conventional angiography is not recommended for the diagnosis of GCA or TAK as it has been superseded by the previously mentioned imaging modalities.

Although conventional angiography has not been included formally as one of the key questions to guide the SLR, the task force felt it was necessary to make a statement. Conventional angiography has been the gold standard for several decades in the diagnosis of LVV, but it is very invasive and involves high resource use and a higher procedural risk as compared with other imaging modalities. Besides, it provides no information about wall morphology, although luminal changes are depicted with detail. The main indication for conventional angiography in LVV is currently as part of vascular interventions such as percutaneous transluminal balloon angioplasty or stenting. ${ }^{40}$

Recommendation 10: in patients with LVV (GCA or TAK) in whom a flare is suspected, imaging might be helpful to confirm 
- To define a gold standard for the diagnosis, particularly of large vessel giant cell arteritis (LV-GCA).

- To directly and prospectively compare the diagnostic value of ultrasound and MRI of cranial arteries for diagnosis of GCA.

- To investigate the diagnostic value of CT of cranial arteries for diagnosis of GCA.

- To compare the value of imaging for the diagnosis of GCA when performed by examiners with low versus high expertise in large vessel vasculitis (LVV) imaging.

- To investigate the additional value of imaging of axillary arteries in all patients with suspected GCA versus performing it in those without a positive imaging of temporal arteries.

- To investigate the value of standardised assessment of different vascular beds by imaging for the diagnosis of GCA.

- To investigate the role of MRI in the diagnosis of GCA in patients with a negative ultrasound.

- To investigate the possible relevance of the assessment of intracranial arteries for the diagnosis and prognosis of GCA.

- To investigate the role of ultrasound, MRI, positron emission tomography (PET) and CT in the diagnosis of LVV with predominantly systemic symptoms.

- To investigate the role of ultrasound, MRI, PET and CT in the diagnosis of Takayasu arteritis.

- To develop tools for the assessment of disease activity in LVV and to agree on definitions of remission and relapse (to better investigate the role of imaging for monitoring of LVV).

- To investigate the additional value of the different imaging modalities in the assessment of disease activity during follow-up over clinical and laboratory assessment alone.

- To investigate the value of imaging (eg, assessment of the extent of vascular involvement) as well as individual vasculitis signs (eg, 'halo' sign, contrast enhancement as compared with wall thickening) as a prognostic factor for LVV outcomes.

- To further study the possibility of differentiating persistent mural inflammation from vascular remodelling (eg, persistent fluorodeoxyglucose uptake in patients in clinical remission).

- To investigate the number of patients needed to screen with imaging methods for identifying cases with aortic complications.

- To define standardised, well-validated scoring methodologies for all imaging modalities and to develop composite scores for imagingbased monitoring of patients with LVV. To compare different imaging modalities for monitoring of aortic complications.

- To study whether therapy should be modified based on imaging results alone.

- To compare therapy modification based on traditional clinical evaluation versus evaluation that includes results of additional imaging.

- To study the value of novel technical developments for diagnosis and monitoring of LVV such as contrast-enhanced ultrasound or PET with ligands specifically targeting immune cells.

or exclude it. Imaging is not routinely recommended for patients in clinical and biochemical remission.

This recommendation is based on expert opinion. In certain situations, for example when clinical and laboratory parameters are inconclusive, imaging may determine the decision whether to change treatment. The primary choice between different imaging modalities depends again on the clinical situation, local availability and expertise. In individual patients, imaging methods might also be complementary, given that the information provided is different (such as local glucose consumption/ metabolism by PET or perfusion by contrast-enhanced imaging).

In patients with a clear-cut clinical flare, as well as in patients in clinical and biochemical remission, the role of additional imaging to determine disease activity is currently unknown.

In one PET study, scans were performed in all newly diagnosed patients with GCA at baseline and during follow-up. ${ }^{41}$ While PET scores significantly dropped from baseline to 3 months, there was no further reduction at 6 months. Up to two-thirds of patients in full clinical remission still revealed a positive PET at both follow-up visits, and PET scores did not significantly differ at times of remission and relapse. Whether ongoing tracer uptake in patients in full clinical remission is caused by low-grade inflammation or remodelling and whether these findings have any impact on future vascular outcomes are issues that have to be clarified by future studies.

Ultrasound studies in GCA reported the disappearance of the ' halo' sign in temporal arteries in the majority of patients after $2-4$ weeks of GC therapy. ${ }^{16} 173442-48$ In extracranial arteries, residual changes often remained visible for several months. The examination of these vessels might be of potential value for monitoring purposes; however, none of these studies addressed whether ultrasound was helpful for the assessment of relapse.

In summary, the limited literature is mainly descriptive and does not add further insights into the additional value of imaging compared with only clinical definition of flare. Further research is urgently needed to address this issue.

Recommendation 11: in patients with LVV (GCA or TAK), MRA, CTA and/or ultrasound may be used for long-term monitoring of structural damage, particularly to detect stenosis, occlusion, dilatation and/or aneurysms. The frequency of screening as well as the imaging method applied should be decided on an individual basis.

The task force suggests, based on expert opinion, that regular screening for structural damage might be performed in GCA and TAK patients with signs or symptoms of stenosis/occlusion or aneurysms, as well as in those with recurrent or persistent inflammation of large arteries and/or the aorta. The choice of the imaging method depends on the vessel(s) affected, local settings and expertise. Monitoring of a patient with inflammation and/or dilatation of the aorta, for example, requires MRI or $\mathrm{CT}$, whereas a stenosis of the axillary/subclavian arteries could be followed up by ultrasound.

The frequency of imaging assessments for vasculitic stenoses should also be decided on an individual basis, as there is currently insufficient data to frame a recommendation. ${ }^{12}$

The development of aortic aneurysms has been reported in patients with GCA despite the absence of ongoing clinical activity. Aortic dilatation might occur even years after disease outset. ${ }^{49}$ Aortic inflammation at baseline as well as male sex, hypertension and smoking history have been described as risk 
factors for aortic dilatation. ${ }^{50} 51$ However, whether and how often imaging of the aorta should be repeated remains an uncertain decision. A chest X-ray and abdominal ultrasound every other year in patients at low risk for aortic aneurysms is current clinical practice in some countries. ${ }^{52}$ However, there are no data demonstrating that such a strategy would have a sufficient sensitivity, specificity and cost-effectiveness. ${ }^{53}$ This is an area that requires further robust research.

Recommendation 12: imaging examination should be done by a trained specialist using appropriate equipment, operational procedures and settings. The reliability of imaging, which has often been a concern, can be improved by specific training. Suggestions for technical and operational parameters are depicted in box 1.

The task force unanimously agreed that the standardisation of investigational procedures as well as the definition of minimal technical and training requirements is essential to produce sensitive, specific and reliable imaging results. ${ }^{15} 54$ The development of specific training programmes as well as national and international courses for imaging in LVV (particularly for ultrasound) should have a high priority in order to facilitate implementation of these recommendations in clinical practice.

The items listed in box 1 are almost entirely based on expert consensus.

No recommendation was made on the prognostic value of imaging modalities in patients with established GCA and TAK because of the absence of evidence and experience. Based on the discussions and the areas of uncertainty, a research agenda has been proposed, which is depicted in box 2 .

\section{DISCUSSION}

These are the first EULAR recommendations providing up-todate guidance for the role of imaging in the diagnosis and monitoring of patients with (suspected) LVV, recognising recent progress in the field. Imaging enables rapid diagnosis of LVV with low burden to patients and should therefore be used as the first diagnostic test provided it is readily available and performed with high quality. To implement the recommendations in clinical practice, training programmes for imaging are required. These principles are reflected in both the recommendations and the research agenda, acknowledging also the gaps in evidence that include direct comparisons of different imaging modalities, the diagnostic value of imaging for predominantly LV-GCA and TAK, as well as the specific value of imaging for monitoring and outcome prediction. Some of the recommendations were mainly based on clinical experience and consensus. Good quality studies are now required to answer the numerous questions raised in the research agenda, so that future recommendations can be upgraded and based on more solid evidence. The present recommendations nevertheless represent a step forward in the approach to patients with (suspected) LVV, and we believe that their implementation will improve patient care.

Previous EULAR recommendations for the management of LVV, already from 2009, recognised the possible value of MRI and PET for the diagnosis and assessment of TAK similar to the present prepositions, whereas for GCA, TAB was previously considered as the only reliable diagnostic test. ${ }^{55}$ The present article is not intended to discredit the role of biopsy as clearly explained in the recommendations; nevertheless, the task force felt that TAB may be dispensable in cases where GCA is confirmed or excluded based on clinical, laboratory and imaging results.

In summary, we developed 12 recommendations on the use of imaging for the diagnosis and monitoring of LVV. These recommendations are supported by evidence along with expert consensus. Unresolved issues and areas of further study have been depicted in the research agenda. We expect that much progress continue to take place in the area of imaging in LVV, and we will carefully follow developments in the field, assuming that an amendment of these recommendations may be needed within a few years.

\section{Author affiliations}

'Department of Rheumatology and Immunology, Medical University Graz, Graz, Austria

${ }^{2}$ Department of Rheumatology, South Tyrol Health Trust, Hospital of Bruneck, Bruneck, Italy

${ }^{3}$ Department of Rheumatology, Leiden University Medical Center, Leiden, The Netherlands

${ }^{4}$ Department of Internal Medicine VI, Medical University Innsbruck, Innsbruck, Austria ${ }^{5}$ Department of Biophysics and Nuclear Medicine, Hôpitaux Universitaires ParisSud, Assistance Publique-Hôpitaux de Paris, Le Kremlin-Bicetre, Île-de-France,

France

${ }^{6}$ IR4M-UMR8081, Université Paris-Saclay, Orsay, France

${ }^{7}$ Department of Diagnostic and Interventional Radiology, University Medical Center Wuerzburg, Wuerzburg, Germany

${ }^{8}$ General Internal Medicine Department, University Hospital Gasthuisberg, Leuven, Belgium

${ }^{9}$ Department of Rheumatology and Clinical Immunology, University Medical Center Groningen, Groiningen, The Netherlands

${ }^{10}$ Research Laboratory and Academic Division of Clinical Rheumatology, Department of Internal Medicine, University of Genova, Genova, Italy

${ }^{11}$ Patient Charity Polymyalgia Rheumatica and Giant Cell Arteritis UK, London, UK

${ }^{12}$ Department of Rheumatology, Southend Hospital NHS Trust, Westcliff-on-Sea, UK

${ }^{13}$ Anglia Ruskin University, Chelmsford, UK

${ }^{14}$ Department of Rheumatology, Martina Hansens Hospital, Baerum, Norway

${ }^{15}$ Division of Rheumatology, Department of Internal Medicine, Marmara University, Istanbul, Turkey

${ }^{16}$ Rheumatology Unit, Universita degli Studi di Torino Dipartimento di Scienze Mediche, Torino, Italy

${ }^{17}$ Polymyalgia Rheumatica and Giant Cell Arteritis Scotland (PMR-GCA Scotland), Perth, UK

${ }^{18}$ Rheumatology Research Unit, Instituto de Medicina Molecular, Faculty of Medicine, University of Lisbon, Lisbon, Portugal

${ }^{19}$ Rheumatology Department, Hospital de Santa Maria - Centro Hospitalar Lisboa Norte, Lisbon, Portugal

${ }^{20}$ Department of Rheumatology, Azienda USL-IRCCS di Reggio Emilia, Reggio Emilia, Italy

${ }^{21}$ Dipartimento di Scienze della Vita, Universita degli Studi di Modena e Reggio Emilia, Reggio Emilia, Italy

${ }^{22}$ Department of Nuclear Medicine and Molecular Imaging, University of Groningen, University Medical Center Groningen, Groningen, The Netherlands

${ }^{23}$ Department of Biomedical Photonic Imaging, University of Twente, Enschede, The Netherlands

${ }^{24}$ Medical Centre for Rheumatology Berlin-Buch, Immanuel Krankenhaus Berlin, Berlin, Germany

Correction notice This article has been corrected since it published Online First. The text in box 1 has been corrected.

Acknowledgements The authors would like to thank Louise Falzon for her work in the development of the literature search strategy, and Alexandre Sepriano for his help with the statistical analysis of the systematic literature review.

Contributors All authors were involved in the discussion and formulation of the recommendations. ChristiaD wrote the first version of the manuscript. All authors reviewed it and made extensive comments and appropriate changes to it. All authors approved the final version of the manuscript.

Funding Funding was provided by the European League Against Rheumatism.

Competing interests $T A B$ received research grants from Deutsche Forschungsgemeinschaft (DFG) and Siemens Healthineers and received consultancies and speaker fees from HeartFlow, GSK, MSD, Roche, Bayer, Bracco, Guerbet and Siemens. EB received consultancies and speaker fees from Roche, which were paid to the University Medical Center Groningen. BD has received consultancies from Roche, GSK and Munidipharma. APD received speaker fees from Roche. WS received consultancies and speakers fee from Roche and GSK. All other authors have no competing interest.

Provenance and peer review Not commissioned; externally peer reviewed.

Data sharing statement No additional data are available. 
(c) Article author(s) (or their employer(s) unless otherwise stated in the text of the article) 2018. All rights reserved. No commercial use is permitted unless otherwise expressly granted.

\section{REFERENCES}

1 Smeeth L, Cook C, Hall AJ. Incidence of diagnosed polymyalgia rheumatica and temporal arteritis in the United Kingdom, 1990-2001. Ann Rheum Dis 2006;65:1093-8.

2 Lawrence RC, Felson DT, Helmick CG, et al. Estimates of the prevalence of arthritis and other rheumatic conditions in the United States. Part II. Arthritis Rheum 2008:58:26-35.

3 Patil P, Williams M, Maw WW, et al. Fast track pathway reduces sight loss in giant cell arteritis: results of a longitudinal observational cohort study. Clin Exp Rheumatol 2015;33.

4 Diamantopoulos AP, Haugeberg G, Lindland A, et al. The fast-track ultrasound clinic for early diagnosis of giant cell arteritis significantly reduces permanent visual impairment: towards a more effective strategy to improve clinical outcome in giant cell arteritis? Rheumatology 2016:55:66-70.

5 Dejaco C, Duftner C, Buttgereit F, et al. The spectrum of giant cell arteritis and polymyalgia rheumatica: revisiting the concept of the disease. Rheumatology 2017:56:506-15.

6 Prieto-González S, Arguis P, Cid MC. Imaging in systemic vasculitis. Curr Opin Rheumatol 2015;27:53-62.

7 Monti S, Floris A, Ponte C, et al. The use of ultrasound to assess giant cell arteritis: review of the current evidence and practical guide for the rheumatologist. Rheumatology 2017 [Epub ahead of print].

8 Direskeneli H. Clinical assessment in Takayasu's arteritis: major challenges and controversies. Clin Exp Rheumatol 2017;35:189-93.

9 van der Heijde D, Aletaha D, Carmona L, et al. 2014 Update of the EULAR standardised operating procedures for EULAR-endorsed recommendations. Ann Rheum Dis 2015;74:8-13.

10 Whiting PF, Rutjes AW, Westwood ME, et al. QUADAS-2: a revised tool for the quality assessment of diagnostic accuracy studies. Ann Intern Med 2011;155:529-36.

11 Hayden JA, Côté P, Bombardier C. Evaluation of the quality of prognosis studies in systematic reviews. Ann Intern Med 2006;144:427-37.

12 Duftner C, Dejaco C, Sepriano A, et al. Imaging in diagnosis, monitoring and outcome prediction of large vessel vasculitis: a systematic literature review and meta-analysis informing the EULAR recommendations. RMD open. submitted.

13 OCEBM Levels of Evidence Working Group. The Oxford 2011 levels of evidence, 2011 http://www.cebm.net/index.aspx?o=5653

14 Salvarani C, Cimino L, Macchioni P, et al. Risk factors for visual loss in an Italian population-based cohort of patients with giant cell arteritis. Arthritis Rheum 2005;53:293-7.

15 Luqmani $R$, Lee $E$, Singh $S$, et al. The role of ultrasound compared to biopsy of temporal arteries in the diagnosis and treatment of giant cell arteritis (TABUL): a diagnostic accuracy and cost-effectiveness study. Health Technol Assess 2016:20:1-238

16 Schmidt WA, Kraft HE, Vorpahl K, et al. Color duplex ultrasonography in the diagnosis of temporal arteritis. N Eng/ J Med 1997:337:1336-42.

17 Karahaliou M, Vaiopoulos G, Papaspyrou S, et al. Colour duplex sonography of temporal arteries before decision for biopsy: a prospective study in 55 patients with suspected giant cell arteritis. Arthritis Res Ther 2006;8:R116.

18 Hauenstein C, Reinhard M, Geiger J, et al. Effects of early corticosteroid treatment on magnetic resonance imaging and ultrasonography findings in giant cell arteritis. Rheumatology 2012:51:1999-2003.

19 Gunawardene AR, Chant $\mathrm{H}$. Facial nerve injury during temporal artery biopsy. Ann $R$ Coll Surg Engl 2014;96:257-60.

20 Germanò G, Muratore F, Cimino L, et al. Is colour duplex sonography-guided temporal artery biopsy useful in the diagnosis of giant cell arteritis? A randomized study. Rheumatology 2015:54:400-4.

21 Schmidt WA, Seifert A, Gromnica-Ihle E, et al. Ultrasound of proximal upper extremity arteries to increase the diagnostic yield in large-vessel giant cell arteritis. Rheumatology 2008;47:96-101

22 Muratore F, Kermani TA, Crowson CS, et al. Large-vessel giant cell arteritis: a cohort study. Rheumatology 2015;54:463-70.

23 Brack A, Martinez-Taboada V, Stanson A, et al. Disease pattern in cranial and largevessel giant cell arteritis. Arthritis Rheum 1999:42:311-7.

24 Griner PF, Mayewski RJ, Mushlin Al, et al. Selection and interpretation of diagnostic tests and procedures. Principles and applications. Ann Intern Med 1981;94:557-92.

25 Rudwaleit M, van der Heijde D, Khan MA, et al. How to diagnose axial spondyloarthritis early. Ann Rheum Dis 2004;63:535-43.

26 Chrysidis S, Duftner C, Dejaco C, et al. Definitions and reliability assessment of elementary ultrasound lesions in giant cell arteritis: a study from the OMERACT Large Vessel Vasculitis Ultrasound Working Group. RMD open;2017. submitted.

27 Aschwanden M, Daikeler T, Kesten F, et al. Temporal artery compression sign-a novel ultrasound finding for the diagnosis of giant cell arteritis. Ultraschall Med 2013:34:47-50
28 Aschwanden M, Imfeld S, Staub D, et al. The ultrasound compression sign to diagnose temporal giant cell arteritis shows an excellent interobserver agreement. Clin Exp Rheumatol 2015;33.

29 Nesher G, Shemesh D, Mates M, et al. The predictive value of the halo sign in color Doppler ultrasonography of the temporal arteries for diagnosing giant cell arteritis. J Rheumatol 2002;29:1224-6.

30 Pfadenhauer K, Weber H. Ultrasonography of the temporal, periorbital and carotid arteries in the diagnosis of giant cell arteritis and its neuroophthalmological complications. Ultraschall Med 2006;27:329-35.

31 Diamantopoulos AP, Haugeberg G, Hetland H, et al. Diagnostic value of color Doppler ultrasonography of temporal arteries and large vessels in giant cell arteritis: a consecutive case series. Arthritis Care Res 2014;66:113-9.

32 Bley TA, Reinhard M, Hauenstein C, et al. Comparison of duplex sonography and high-resolution magnetic resonance imaging in the diagnosis of giant cell (temporal) arteritis. Arthritis Rheum 2008:58:2574-8.

33 Siemonsen S, Brekenfeld C, Holst B, et al. 3T MRI reveals extra- and intracranial involvement in giant cell arteritis. AJNR Am J Neuroradiol 2015:36:91-7.

34 Aschwanden M, Kesten F, Stern M, et al. Vascular involvement in patients with giant cell arteritis determined by duplex sonography of $2 \times 11$ arterial regions. Ann Rheum Dis 2010;69:1356-9.

35 Lariviere D, Benali K, Coustet B, et al. Positron emission tomography and computed tomography angiography for the diagnosis of giant cell arteritis: A real-life prospective study. Medicine 2016;95:e4146.

36 Blockmans D, Stroobants S, Maes A, et al. Positron emission tomography in giant cell arteritis and polymyalgia rheumatica: evidence for inflammation of the aortic arch. Am J Med 2000;108:246-9.

37 Puppo C, Massollo M, Paparo F, et al. Giant cell arteritis: a systematic review of the qualitative and semiquantitative methods to assess vasculitis with 18F-fluorodeoxyglucose positron emission tomography. Biomed Res Int 2014:2014:1-11.

38 Yamada I, Nakagawa T, Himeno Y, et al. Takayasu arteritis: diagnosis with breathhold contrast-enhanced three-dimensional MR angiography. J Magn Reson Imaging 2000;11:481-7.

39 Yamada I, Nakagawa T, Himeno Y, et al. Takayasu arteritis: evaluation of the thoracic aorta with CT angiography. Radiology 1998;209:103-9.

40 Gülcü A, Gezer NS, Akar S, et al. Long-term follow-up of endovascular repair in the management of arterial stenosis caused by takayasu's arteritis. Ann Vasc Surg 2017:42:93-100

41 Blockmans D, de Ceuninck L, Vanderschueren S, et al. Repetitive 18F-fluorodeoxyglucose positron emission tomography in giant cell arteritis: a prospective study of 35 patients. Arthritis Rheum 2006:55:131-7.

42 De Miguel E, Roxo A, Castillo C, et al. The utility and sensitivity of colour Doppler ultrasound in monitoring changes in giant cell arteritis. Clin Exp Rheumatol 2012;30:\$34-8.

43 Habib HM, Essa AA, Hassan AA. Color duplex ultrasonography of temporal arteries: role in diagnosis and follow-up of suspected cases of temporal arteritis. Clin Rheumatol 2012:31:231-7.

44 Pérez López J, Solans Laqué R, Bosch Gil JA, et al. Colour-duplex ultrasonography of the temporal and ophthalmic arteries in the diagnosis and follow-up of giant cell arteritis. Clin Exp Rheumatol 2009;27:S77-82.

45 Pfadenhauer K, Weber H. Duplex sonography of the temporal and occipital artery in the diagnosis of temporal arteritis. A prospective study. J Rheumatol 2003;30:2177-81.

46 Salvarani C, Silingardi M, Ghirarduzzi A, et al. Is duplex ultrasonography useful for the diagnosis of giant-cell arteritis? Ann Intern Med 2002;137:232-8.

47 Schmidt WA, Moll A, Seifert A, et al. Prognosis of large-vessel giant cell arteritis. Rheumatology 2008;47:1406-8.

48 Schmidt WA, Natusch A, Möller DE, et al. Involvement of peripheral arteries in giant cell arteritis: a color Doppler sonography study. Clin Exp Rheumatol 2002;20:309-18.

49 García-Martínez A, Arguis P, Prieto-González S, et al. Prospective long term follow-up of a cohort of patients with giant cell arteritis screened for aortic structural damage (aneurysm or dilatation). Ann Rheum Dis 2014;73:1826-32

50 Blockmans D, Coudyzer W, Vanderschueren S, et al. Relationship between fluorodeoxyglucose uptake in the large vessels and late aortic diameter in giant cel arteritis. Rheumatology 2008:47:1179-84

51 Robson JC, Kiran A, Maskell J, et al. The relative risk of aortic aneurysm in patients with giant cell arteritis compared with the general population of the UK. Ann Rheum Dis 2015;74:129-35

52 Dasgupta B, Borg FA, Hassan N, et al. BSR and BHPR guidelines for the management of giant cell arteritis. Rheumatology 2010;49:1594-7.

53 Mackie SL, Hensor EM, Morgan AW, et al. Should I send my patient with previous giant cell arteritis for imaging of the thoracic aorta? A systematic literature review and meta-analysis. Ann Rheum Dis 2014;73:143-8.

54 De Miguel E, Castillo C, Rodríguez A, et al. Learning and reliability of colour Doppler ultrasound in giant cell arteritis. Clin Exp Rheumatol 2009:27:S53-8.

55 Mukhtyar C, Guillevin L, Cid MC, et al. EULAR recommendations for the management of large vessel vasculitis. Ann Rheum Dis 2009;68:318-23. 